\title{
SHEETS OF CONJUGACY CLASSES IN SIMPLE ALGEBRAIC GROUPS
}

\author{
IULIAN I. SIMION
}

\begin{abstract}
For a connected reductive algebraic group $G$ defined over an algebraically closed field of characteristic $p$ the sheets of conjugacy classes have been parametrized by G. Carnovale and F. Esposito when $p$ is good for $G$. We show that the method is independent of characteristic and that a similar parametrization is possible for all $p$.
\end{abstract}

MSC 2010. 14L10, 14L35, 14L40.

Key words. Algebraic groups, Jordan classes, sheets of conjugacy classes.

\section{REFERENCES}

[1] A. Borel, Linear Algebraic Groups, Graduate Texts in Mathematics, Vol. 126, Springer Verlag, New York, second edition, 1991.

[2] W. Borho, Über Schichten halbeinfacher Lie-Algebren, Invent. Math., 65 (1981), 283-317.

[3] G. Carnovale and F. Esposito, On sheets of conjugacy classes in good characteristic, Int. Math. Res. Not. IMRN, 4 (2012), 810-828.

[4] R. W. Carter, Finite groups of Lie type - Conjugacy classes and complex characters, John Wiley \& Sons Ltd., Chichester, 1993.

[5] D. I. Deriziotis, The Brauer complex of a Chevalley group, J. Algebra, 70 (1981), 261-269.

[6] D. I. Deriziotis, Centralizers of semisimple elements in a Chevalley group, Comm. Algebra, 9 (1981), 1997-2014.

[7] W. Hoffmann, Induced conjugacy classes, prehomogeneous varieties, and canonical parabolic subgroups, arXiv:1206.3068 [math.GR], 2012.

[8] J. E. Humphreys, Conjugacy classes in semisimple algebraic groups, Mathematical Surveys and Monographs, Vol. 43, Amer. Math. Soc., Providence, RI, 1995.

[9] M. W. Liebeck and G. M. Seitz, Unipotent and nilpotent classes in simple algebraic groups and Lie algebras, Mathematical Surveys and Monographs, vol. 180, Amer. Math. Soc., Providence, RI, 2012.

[10] G. Lusztig and N. Spaltenstein, Induced unipotent classes, J. Lond. Math. Soc., 19 (1979), 41-52.

[11] G. Lusztig, On the finiteness of the number of unipotent classes, Invent. Math., 34 (1976), 201-213.

[12] G. Lusztig, Intersection cohomology complexes on a reductive group, Invent. Math., 75 (1984), 205-272.

[13] G. Malle and D. Testerman, Linear algebraic groups and finite groups of Lie type, Cambridge Studies in Advanced Mathematics, Vol. 133, Cambridge University Press, Cambridge, 2011.

DOI: $10.24193 /$ mathcluj.2019.2.08 
[14] G. J. McNinch and E. Sommers, Component groups of unipotent centralizers in good characteristic, J. Algebra, 260 (2003), 323-337.

[15] N. Spaltenstein, Classes unipotentes et sous-groupes de Borel, Lecture Notes in Mathematics, Vol. 946, Springer Verlag, Berlin, 1982.

[16] T. A. Springer, Some arithmetical results on semi-simple Lie algebras, Publ. Math. Inst. Hautes Études Sci., 30 (1966), 115-141.

[17] T. A. Springer and R. Steinberg, Conjugacy classes, in Seminar on Algebraic Groups and Related Finite Groups, Lecture Notes in Mathematics, Vol. 131, Springer, Berlin, 1970, pp. 167-266.

[18] R. Steinberg, Conjugacy classes in algebraic groups, Lecture Notes in Mathematics, Vol. 366, Springer Verlag, Berlin, 1974.

Received June 24, 2018

Babes-Bolyai University

Accepted August 3, 2018

Faculty of Mathematics

and Computer Science

1 M. Kogălniceanu St.

400084 Cluj-Napoca

E-mail: simion@math.ubbcluj.ro 